\title{
Investigación en la Escuela Normal Superior*
}

\author{
Hilda Mar Rodríguez Gómez ${ }^{1}$
}

\section{Resumen}

Las dificultades existentes en el proceso de formación de maestros pueden ser superadas a través de diferentes estrategias de trabajo colaborativo, que permitan pensar la acción del maestro y su formación como un espacio propio de la pedagogía, cruzado de manera orgánica por saberes, disciplinas y ciencias que contribuyen a concebir el maestro como un intelectual de la pedagogía, como sujeto de saber pedagógico y como formador. Para ello, se propone la investigación en el aula y el método de proyectos como una alternativa de construcción y reconstrucción de la cotidianidad a partir de la relación entre la práctica pedagógica y la investigación educativa.

Palabras clave: Formación docente, trabajo colaborativo, práctica pedagógica

\section{Summary}

The current difficulties in the teacher training process can be overcome through different strategies of collaborative work. They could allow seeing teacher's action and training as a natural place for pedagogy, crossed in an organic way for knowledge, disciplines and sciences which may as well help teachers to be conceived as intellectuals of pedagogy, subjects of pedagogical knowledge and trainers. That's the reason why school research and project method are proposed as an alternative of construction and reconstruction of the everyday from the existent relationship between pedagogical practice and educational research.

Key words: Teacher's training, collaborative work, pedagogical practice

\section{Presentación}

Las alternativas que se proponen desde el proyecto Aciforma ${ }^{2}$ pretenden superar los problemas que soporta el proceso de formación del maestro -uno de los cuales es la investigación- y pensarlo como un espacio propio de la pedagogía, cruzado de manera orgánica por saberes, disciplinas y ciencias que contribuyen a concebir el maestro como un intelectual de la pedagogía, como sujeto de saber pedagógico y como formador. Se pretende formar un maestro crítico, capaz de hacer de su trabajo una rutina ${ }^{3}$ intelectual dentro del campo conceptual de la pedagogía, con habilidades para comprender las transformaciones de las situaciones pedagógicas y, en éstas, crear, producir e innovar. En

\footnotetext{
* Texto recibido el 27 de julio de 2004 y arbitrado el 26 de agosto de 2004.

1 Profesora del Departamento de Educación Médica de la Facultad de Medicina de la Universidad de Antioquia, investigadora del grupo Diverser, en pedagogía y sistemas simbólicos. Coinvestigadora en el grupo de Historia de la Práctica Pedagógica. E-mail: hildamar98@yahoo.com

2 Apropiación pedagógica del campo intelectual de la educación en Colombia para la formación de docentes, Proyecto de investigación dirigido por el profesor Alberto Echeverri, miembro fundador del grupo Historia de las Prácticas Pedagógicas en Colombia, fue financiado por Colciencias, la Universidad de Antioquia y la Secretaría de Educación y Cultura de Antioquia, y se realizó entre los años 1996-2002.

${ }^{3}$ Se emplea el término "rutina" en el mismo sentido que la psicología cognitiva lo refiere al conocimiento de los expertos, conocimiento altamente codificado.
} 
definitiva, se trata de formar un maestro capaz de experimentar su saber, validar su conocimiento en el interior de sus acciones y acceder a la construcción de su propia práctica pedagógica. En este texto, se restringe la reflexión a la práctica pedagógica y su relación con la investigación, no como la medida del maestro, el límite de su espacio o la máxima expresión de la actividad escolar, sino como práctica que se instala en la búsqueda de aquellos fundamentos que le permitan ubicarse en una dimensión diferente de la enseñanza, a la vez que articular alrededor de este concepto nociones que hacen de la práctica pedagógica un ejercicio del pensamiento.

\section{El proyecto Aciforma y sus conceptos: camino para la interpretación de la investigación}

El proyecto de investigación Aciforma buscó vincular a los maestros de las Escuelas Normales Superiores a una nueva experiencia del tiempo y el espacio pedagógico, la cual intentaba llevar al maestro más allá de las paredes de la escuela al darle la posibilidad de experimentarse en la instalación de proyectos en la ciudad, la sociedad y la imagen. Esta experiencia buscaba dotar a las instituciones formadoras de un nuevo horizonte para definir los límites entre el interior y el exterior, el afuera y el adentro; en síntesis, con el entorno, trátese de la cultura, la etnia, lo público o lo ecológico.

Una institución formadora, incapaz de interpretar pedagógicamente los dolores y las angustias de sus gentes y los maltratos de la naturaleza no puede llamarse Escuela Normal Superior. El desarrollo de una sensibilidad hacia la exterioridad se buscó mediante el acercamiento del profesorado a procesos como la construcción del equipo docente, del proyecto general de investigación y de los proyectos de núcleo y aula, elementos presentes en los seminarios permanentes.

Venimos de una tradición en donde el encierro hace olvidar la exterioridad, y donde el manual y el texto atrapan al profesor y al estudiante en el aula, de la misma manera que los Aurelianos ${ }^{4}$ y sus descendientes pasaban los días, los meses y los años en el laboratorio de alquimia, aislados del mundo circundante.

Nuestra labor ha sido proponer ámbitos, espejos, contextos donde nosotros y los maestros nos podamos dibujar y borrar nuestros rostros, comenzar y recomenzar. Algunos de estos espejos están constituidos por conceptos.

Una mirada a todos aquellos conceptos que el proyecto Aciforma produjo a lo largo de sus seis años, permite identificar el marco de referencia y la guía para la orientación en el campo conceptual de la pedagogía, sirve además para comprender las implicaciones de la investigación pedagógica en la Escuela Normal Superior.

\section{Los conceptos}

La no existencia de un campo de formación específico que permita la apropiación y producción de los saberes, experiencias e innovaciones que se producen en el terreno de la educación, es una de las dificultades con que tropieza hoy día la formación de maestros en nuestro país. Por ello, la contribución de Echeverri (2000) al formular un campo conceptual de la pedagogía (CCP), que regula las relaciones con el exterior a partir de los conceptos articuladores y hacia el interior a través del análisis de los diferentes proyectos

\footnotetext{
${ }^{4}$ Me refiero a los personajes principales de la obra del premio Nobel colombiano, Gabriel García Márquez, Cien años de soledad. 
reconfiguradores, para producir una aproximación desde la cual sea posible comprender la formación de maestros como un proceso de largo aliento que supera las fragmentaciones producidas por instituciones como la escuela anexa o modelos y dispositivos desagregantes de la perspectiva pedagógica de la formación de maestros. La construcción del CCP es especialmente significativa, dado que se propone incluir o insertar las teorías, los conceptos, las experiencias, experimentaciones, experiencias e innovaciones en un espacio conceptual que goza de autonomía y, a la vez, de capacidad relacional. Para comprender lo que el CCP significa, puede hacerse a través del dispositivo formativo comprensivo (DFC) como una opción para desplegar la formación y la comprensión sobre la práctica pedagógica, de modo que sea posible la producción de experiencias educativas, la construcción de espacios propios para la subjetivación del maestro, al tiempo que permite la pedagogización de los espacios de la institución, entendiendo por esto la tematización, desde la pedagogía -referentes formativos, investigación, práctica, campo aplicado- de los espacios cotidianos de la Escuela Normal Superior. Se trata de la elaboración reflexiva de información pedagógica necesaria para el ejercicio de la enseñanza, el diseño y la experimentación de innovaciones y la elaboración de nociones alrededor de los conceptos articuladores, los referentes formativos y los proyectos de reconfiguración.

En otras palabras, el DFC es una compleja red que lleva el campo conceptual de la pedagogía a las instituciones formadoras de maestros, en la medida en que pone a circular la naturaleza formativa de éstas, manifiesta en la consideración de la totalidad de relaciones que se establecen entre las Escuelas Normales y la ciencia, la tecnología y la cultura. Así, la Escuela Normal convierte en objeto de investigación su oficio o naturaleza: la formación de maestros.

De este modo, el dispositivo modifica y transforma las relaciones intersubjetivas dentro de la institución en el espacio y en el tiempo, al recoger, anudar y ordenar en una red los elementos,las situaciones y los acontecimientos que multiplican los sentidos, y los dirige hacia lo formativo y lo comprensivo. Esto se verifica en las relaciones que se establecen en la Normal, y puestas en circulación a través de dos agrupamientos humanos, intelectuales, de saber, que se gestan como condición de esta misma naturaleza lábil, múltiple y compleja: los núcleos y el equipo docente.

El núcleo y el equipo docente en la Escuela Normal Superior no sólo cumplen funciones de reunión o conjunción de profesores. Su papel principal se inscribe en la lógica de un espacio interdisciplinario de reflexión en el cual es posible la apropiación, difusión y construcción de conocimiento relacionado con los saberes y la relación de éstos con el campo conceptual de la pedagogía. Estos encuentros colectivos son la forma de operacionalizar la reflexión sobre los conceptos articuladores y sus niveles de funcionamiento (observación, experimentación, conceptualización y teorización). La importancia de este encuentro reflexivo radica en la necesidad de dotar a la institución normalista de elementos.

Los conceptos articuladores deben entenderse como aquellos que -en relación con la tradición pedagógica- denotan la capacidad de las instituciones normalistas para apropiar y producir desplazamientos de sus sentidos en los niveles de funcionamiento y en relación con las funciones que cumplen. Los conceptos articuladores son la conciencia pedagógica de la Normal que circula en el espacio de formación. Habitan el campo aplicado en el despliegue de sus funciones (de regulación, traducción, prescripción y producción de los conceptos).

Los elementos enunciados previamente: el dispositivo formativo comprensivo, los núcleos, el equipo docente y los conceptos articuladores se disponen en una estructura móvil y 
dinámica que posibilita que la investigación en el aula y la constitución de un maestro investigador sean una realidad a través de la creación de un espacio que conjugue la experiencia y la innovación: el campo aplicado, que es la materialización de aquellos elementos relacionados con el dispositivo formativo comprensivo (DFC): los núcleos, los conceptos articuladores, los proyectos de investigación, los referentes formativos, la tradición crítica de la pedagogía. En fin, el campo aplicado es la posibilidad de conjugar los aspectos del DFC en una propuesta de regulación, organización, evaluación y producción de todos aquellos aspectos relacionados con la experiencia, la experimentación y la investigación en la Escuela Normal Superior. Su función principal, además de la constitución de la práctica y la difusión de los sentidos de ésta, es la de ejercer críticamente sobre todos aquellos aspectos que configuran y se generan a partir de la práctica. Esto se hace posible a través de la consideración del concepto de enseñanza como una práctica de saber, que inscrita en las realizaciones del enseñar, permite la legitimación de las acciones emprendidas, al tiempo que hace posible la recuperación del papel activo del maestro en la construcción del conocimiento pedagógico.

De lo que se trata en el campo aplicado es de retomar la experiencia como el espacio en el que los referentes formativos y los conceptos articuladores producen sentidos y significados que se conjugan e insertan con estructuras móviles, cambiantes y complejas -como las de la investigación- produciendo en este movimiento un tejido y un espacio en el cual se configura la práctica pedagógica. La experiencia y la investigación dentro del dispositivo permiten al maestro -en tanto posibilita su organización en complejos y redesun mayor desarrollo pedagógico, didáctico y científico, pues lo inscribe en una lógica y una estructura institucional cruzada por el equipo docente y sus tejidos de acción y reflexión. El campo aplicado ofrece alternativas, teóricas y experimentales, para comprender la investigación en el aula y para construir experiencias de carácter educativo y pedagógico que permitan la transformación de la enseñanza y dinamicen el trabajo del maestro.

Los conceptos producidos en el proyecto nos dotan de una capacidad relacional para comprender la formación de maestros y maestras desde diversos ejes, entre los que la investigación se cuenta como una opción de reconstrucción y afirmación intersubjetiva del ser pedagógico del maestro.

\section{Las transformaciones del sistema educativo y la investigación}

Las perspectivas que se abren con la reforma del sistema educativo hacen imprescindible racionalizar y planificar todos aquellos aspectos que posibilitan el cambio. En este sentido, la investigación educativa constituye un campo de actuación fundamental.

La mejora de la calidad de la enseñanza que la sociedad reclama a la educación depende, en buena medida, de la eficacia en los avances de la investigación educativa, y de la generalización e incorporación, a la labor docente, de los aportes de esta investigación, así como de la participación del maestro en proyectos de investigación íntimamente ligados a su práctica cotidiana.

La transformación de la realidad de las instituciones educativas es un proceso de largo aliento que exige constancia y deseo de transformar, teniendo como referente constante la tradición, entendida como la pervivencia de los logros máximos de una teoría o producción. Por ello se hace necesario buscar los fundamentos del cambio en los legados históricos que la pedagogía ha dejado a sus lectores y pensadores, así como en los aportes que las nuevas tendencias han ido plasmando en el campo aplicado. 
La presente propuesta intenta combinar los elementos que aportan a la configuración de la práctica y a su desarrollo desde una perspectiva investigativa: la que ofrece el método de proyectos, que es la opción que permite la construcción de maestros investigadores en el marco de la construcción cooperativa del conocimiento y del intercambio de ideas y opiniones. La investigación en el aula debe partir y fortalecer el equipo docente.

Para incrustar la propuesta del método de proyectos en el dispositivo formativo comprensivo es necesario hacerlo a través de lo que la investigación en el aula significa y el modo como la investigación, a través de los proyectos del núcleo, se despliega por la institución normalista, haciendo de ésta una institución que investiga con el propósito de crear condiciones para la existencia del campo aplicado, es decir, para la experimentación.

\section{Investigación educativa}

En la investigación educativa, y de acuerdo con lo que plantea Eisner (1998), la tradición en investigación, indagación para el autor, es un punto medio entre la investigación y la evaluación cualitativa y su relación con el ver, como aspecto básico del hacer, de la acción del maestro, del equipo docente. Y es que el ver, la mirada, el ojo ilustrado es un mecanismo, dispositivo o contenido de la investigación como forma de ver y comprender el mundo, una manera de operar sobre el mundo.

La investigación en las Escuelas Normales Superiores, además de partir del equipo docente y de los núcleos, de dirigirse al aula y transformar las prácticas pedagógicas, ha de centrarse en el marco amplio de la vida institucional; de allí se desprende que la naturaleza o características de la investigación no se derivan de las formas o procedimientos empleados, sino de la amplitud de su campo de acción, que permite la construcción de respuestas significativas en el entorno inmediato.

Para fortalecer las reflexiones que desde el DFC se realizan en torno a la investigación es necesario definir el fundamento de la investigación en la Normal, como la capacidad de vincular toda la institución de manera ordenada, a través de un tejido que se verifica desde el equipo docente hacia el campo aplicado. Se trata de un flujo de relaciones multidireccionales que acogen en su proceso todos los espacios, referentes, proyectos, realidades, prácticas y sentidos de la Escuela Normal Superior.

Desde el dispositivo formativo comprensivo, la investigación es además una forma de superar el asignaturismo, de volverse sobre el tiempo pedagógico y hacer de éste una realidad formativa, de conjugar las diversas realidades de la institución y anudarlas a una propuesta pedagógica que no dispersa los esfuerzos de la institución. En palabras de Elliot (1993), y refiriéndose a la investigación-acción, para la que también emplea el término práctica reflexiva como ciencia moral:

La investigación-acción integra enseñanza y el desarrollo del profesor, desarrollo del curriculum y evaluación, investigación y reflexión filosófica en un concepto unificado de práctica reflexiva educativa. Esta concepción unificada tiene consecuencias de poder en la medida en que rechaza una división de trabajo rígida en donde las tareas y roles especializados se distribuyen en actividades organizadas desde un punto de vista jerárquico (73).

De este modo, la investigación va a ser el lugar desde el cual habla y actúa la institución. Se superan con esto los modelos fragmentarios y artificiales que piensan la investigación educativa como un implante en la vida institucional, que se verifica en ciertas superficies 
sin que la cotidianidad se afecte o se involucre; y se accede a su formulación desde la misma naturaleza formativa de la Normal, desde la conjunción del equipo docente, como eje articulador de la Normal, como lugar de construcción y confrontación colectiva. Pues se trata, a través de la investigación y del equipo docente, de saltar la barrera de la soledad para acceder a espacios de colegialidad, porque al compartir en el contexto de una investigación cooperativa, los profesores podrían desarrollar grandes elementos comunes de conocimientos profesionales, cultura colectiva que ayudaría a pensar constructivamente a cada profesor individual y que, a su vez, se vería reforzada por las contribuciones de todos. Aunque cada profesor puede mejorar su ejercicio docente por su cuenta, necesita poder acceder al conjunto de conocimientos comunes generados mediante el intercambio y la discusión de las anotaciones e informes de todos, si no quieren "volver a descubrir el Mediterráneo" (Elliot, 178-179).

\section{Formación en investigación educativa (EI)}

La formación en investigación no puede ofrecerse al margen de una consolidación del saber pedagógico del maestro, pues aquél servirá como referente de las acciones investigadoras de los maestros, de modo tal que sea posible la contextualización y reconceptualización de los hallazgos de investigación. Por ello, es necesario fortalecer la formación pedagógica de los maestros y, a la luz de ella, iniciar su proceso de formación en investigación, teniendo claro que ésta debe darse en forma paralela con investigaciones concretas, producto de la acción del equipo docente.

La formación de un maestro investigador, y su acción dentro de las instituciones educativas, requieren que lo incite a buscar respuesta a las preguntas e inquietudes que la práctica diaria le formulan, y a la conformación de grupos de trabajo, que se conviertan en comunidades de investigación.

\section{DFC, IE y Escuela Normal}

Para el caso de la Escuela Normal Superior, la investigación debe permitir la mejora de la enseñanza y la reforma escolar, además de propiciar la escritura de los maestros, la construcción de narraciones, porque la narración permite un acceso diferente a la realidad, a los mundos que se construyen mediante el lenguaje, que son, en última instancia, los mundos que se conocen. La narración abre paso al diario pedagógico, que desde la tradición pedagógica, el dispositivo formativo comprensivo y el campo aplicado busca responder a preguntas relacionadas con la observación y la reflexión, la experiencia y la experimentación.

La investigación educativa debe dejar de ser una actividad de dedicación exclusiva de los especialistas, para convertirse en un acto cotidiano en el que estén implicados los maestros, dado que la intención inicial debe ser tanto la transformación de la práctica educativa, como la producción de conocimiento en torno a la pedagogía y la didáctica.

Así, la enseñanza en la Escuela Normal Superior, a la vez que se inicia allí, posibilita el equipo docente, los núcleos, la investigación en el aula y la innovación pedagógica. En resumen, la investigación es uno de los mecanismos a través de los cuales la Normal se vuelve sobre sí misma y se despliega hacia el exterior para hacer de la formación un principio de acción. 
La formación en la investigación educativa debe proveer al maestro de los elementos necesarios para reconsiderar su práctica, reconocer hechos o sucesos dignos de consideración, teorizar al respecto, llevar registros adecuados de cada una de las etapas de la investigación, e iniciar al maestro en el uso de técnicas e instrumentos de investigación que lleven a una cualificación de su oficio. También la investigación, dentro de la institución normalista, apunta a la producción de conocimiento pedagógico y didáctico que haga realidad las innovaciones en el aula de clase, y a la multiplicación de los espacios de formación; es decir, a la producción de campos aplicados que posibiliten el despliegue del dispositivo formativo comprensivo, y en esa medida contribuya a la transformación del tiempo y el espacio.

La investigación tiene como reto, además de los ya mencionados anteriormente, orientar la construcción de una nueva espacialidad y temporalidad. Es decir, que el tiempo y el espacio de la Escuela Normal Superior deben ser instancias que trascienden lo burocrático y rescaten lo pedagógico. En primer lugar, se deben fomentar experiencias que modifiquen el acontecer en el aula de clase, que generen variaciones en el método de enseñanza. En segundo lugar, se busca borrar las fronteras entre lo interior y lo exterior, de modo que la multiplicación de los campos aplicados sea una esfera de acción en la que el profesor se libera de las ataduras convencionales de trabajo, y accede a formas alternas que se caracterizan por el trabajo cooperativo. El maestro se asume en el equipo docente, y desde éste rescata el tiempo de la enseñanza, que es el tiempo de la pregunta, el tiempo de la inquietud, el tiempo de la investigación. Pero éste sólo se genera a partir del tiempo escolar (el tiempo del enseñar) en el que el maestro se vive como enseñante. La conjunción de estos dos tiempos, además de permitir la investigación, posibilita la experiencia de sí, de maestro, su experiencia de saber y de sujeto de saber.

\section{Investigación en el aula}

La investigación en el aula es un proceso de construcción y reconstrucción de la cotidianidad del aula, a través del reconocimiento y valoración del saber del maestro. Por ello, es necesario analizar las diferentes formas que toma el discurso del maestro en el aula, considerando aspectos como: el lenguaje, las relaciones entre los diferentes actores del sistema educativo y los modos de producción de estos actores en los contextos que conforman la vida escolar (el aula de clase, el recreo, las reuniones de profesores, los rituales de disciplina).

Los comienzos de la investigación en el aula son simples, comunes; parten de preguntas que colman de sentido la acción del maestro y de los alumnos. La investigación en el aula es un espacio que se construye día a día en las interacciones y las conjugaciones de los saberes (explícitos o no) que posee el maestro. La investigación en el aula es un camino para transformar la enseñanza, para modificar los lugares de la escuela, para dinamizar la vida e incluir en ella el saber que proviene de la comunidad; igualmente, es una oportunidad para recoger las dudas y otorgarles un sentido nuevo, distinto, dentro de la dinámica de la acción del maestro.

Con la investigación en el aula, se trata, al igual que con la investigación educativa en general, de desprender ésta de la vida institucional, de las condiciones de existencia del maestro, del núcleo y del equipo docente. Se trata de descentrar la función del maestro de la enseñanza -en sentido instrumental- y ponerla a girar alrededor de la investigación. Con este proceso se logra atenuar el énfasis puesto por las instituciones formadoras en aquellos aspectos externos al maestro, pudiéramos decir aledaños, elementos que bordean la constitución del maestro como sujeto de saber pedagógico, pero que no tienen 
una incidencia directa en su estructura. EI DFC, al plantear la investigación como eje de la institución normalista, busca develar la naturaleza pedagógica de los procesos formativos y recuperar su interioridad.

\section{Para García (1995):}

La investigación en el aula es el proceso por medio del cual los docentes en ejercicio producen una argumentación sistemática de la praxis con las intencionalidades y autodeterminaciones propias de la profesión. Si bien la investigación en el aula es un proceso particular que no puede confundirse con otros, hay que dejar establecido de una vez que dicho proceso forma parte inherente de la enseñanza y no puede definirse como una superestructura ajena cuya existencia provenga de otras dimensiones (79).

Debe quedar claro, entonces, que la investigación en el aula no es un método, no se trata de una técnica que pueda aplicarse en lugar de otra con el fin de mejorar la práctica, tampoco es el encerramiento en el aula para ensayar formas, métodos o estrategias. Se trata, más bien, de una forma de reflexionar sobre aquellos aspectos que en la interioridad del espacio de la enseñanza suceden y ocurren, es "(...) una mirada investigativa. No es ésta una mirada objetivante, sino por el contrario, una mirada reflexiva y personalizante que permite percibir situaciones y relaciones antes ocultas y despersonalizadas por la rutina" (Vasco, 1995: 53).

La investigación en el aula es la posibilidad de problematizar las situaciones cotidianas y hacer de ellas temáticas, a través de las cuales se puede generar conocimiento pedagógico y didáctico, de modo tal que sea posible la reconceptualización de las acciones, conceptos y teorías que están circulando, en la superficie de la escritura, a la luz de los conceptos articuladores, de los referentes formativos y los proyectos de reconfiguración.

Empleando algunos planteamientos de Carr y Kemmis (1988), es posible caracterizar la investigación en el aula como sigue:

(...) En primer lugar, es en sí misma un proceso histórico de transformación de prácticas, de entendimiento y de situaciones: tiene lugar en la historia y a través de ella. (...) En segundo lugar, (...) implica relacionar las prácticas, los entendimientos y las situaciones entre sí; es decir, descubrir correspondencias o ausencia de correspondencias entre entendimientos y prácticas (...) entre prácticas y situaciones (...) así como entre entendimientos y situaciones. (...) (193).

La investigación en el aula es realizada por maestros en ejercicio, y se propone, además de transformar las condiciones de espacio y tiempo en la Escuela Normal, permitir el desarrollo de los proyectos-núcleo, fortalecer el equipo docente -como comunidad pedagógica de investigación-, transformar la práctica, en la medida en que ésta recoge nuevas formas de conducirse y orientarse que introducen reorientaciones y nuevos sentidos en ella; igualmente, los resultados que produce, y que son sistematizados en el diario pedagógico, pueden considerarse como la construcción de argumentos, ideas, hipótesis, experiencias que van a alimentar el campo pedagógico.

La investigación en el aula es un proceso de construcción y reconstrucción de la cotidianidad del aula, a través del reconocimiento y valoración del saber del maestro, desde una perspectiva interactiva e integrada, es un espacio que se construye día a día en las interacciones y las conjugaciones de los saberes (explícitos o no) que posee el maestro; es un camino para transformar la enseñanza, para modificar el espacio de la Normal, para dinamizar la vida e incluir en ella el saber que proviene de la comunidad; es 
una posibilidad que se ofrece el maestro de ser enseñante e investigador, convirtiendo su acción en un doble tejido que cubre el proceso de formación de maestros.

El sentido de estas líneas se comprende en la medida en que la investigación -educativa o en el aula- debe partir de las consideraciones que hace el DFC en torno al maestro, en tanto es fundamental que tenga un espacio, un lugar desde donde pensar, hablar y actuar en el mundo del saber y el conocimiento. Es decir, la construcción -y posterior despliegue- del maestro y de la Escuela Normal Superior debe hacerse de adentro hacia afuera. En esa medida, la investigación es un proceso que parte de las cosas que el maestro conoce, de aquellos elementos, estructuras, fundamentos o ideas con las que ha estado en relación desde el origen mismo de su experiencia.

La investigación en el aula parte, pues, de las preguntas, inquietudes y experiencias que el maestro, el núcleo y el equipo docente tienen sobre la enseñanza, el aprendizaje, la formación, la instrucción, la educación; en fin sobre los conceptos articuladores ${ }^{5}$. Una vez instalado allí, puede empezar la construcción de relaciones con los referentes formativos, de modo que pueda conjugar todas las demandas que se le plantean en torno a la formación desde la perspectiva de lo formativo.

Desde la investigación en el aula se trata de potenciar las miradas sobre la enseñanza y sus articulaciones a la vida del sujeto y la institución, de observar el campo aplicado, y a partir de él producir conocimiento en el paso de una experiencia-actividad, suma de acciones, a una experiencia-reflexión e investigación, en tanto lo experiencial no será ya definido por lo meramente observado sino por las relaciones que configuran el plan. La organización del saber agudiza las preguntas, despliega nuevas relaciones y permite la comprensión en la medida en que hace aparecer la pertinencia del plan elaborado y su aplicación experiencial. De este modo la comprensión se acompaña de la explicación (Palacio y Salinas, 1996)

En síntesis, de lo que se trata en la investigación en el aula es de forjar el campo aplicado, como campo de experimentación e investigación pedagógica, "sobre registros que vinculen la escritura a la tradición escrita, y ésta a la cotidianidad del maestro y el entorno cultural" (Echeverri, 1996). Es un intento de unir enseñanza e investigación, a través de la experimentación, como forma de legitimar la naturaleza formativa de la Escuela Normal Superior.

\section{El método de proyectos ${ }^{6}$}

Representa este nuevo proceder en educación una protesta contra el intelectualismo, el verbalismo y el memorismo que apagaron la curiosidad por todo lo que no fuera el libro, única fuente de verdad. Una educación de espaldas a las lecciones del mundo exterior tuvo que engendrar una rutina y un relajamiento que hacían imposible adaptarse a la evolución que se operaba en la vida y que cegaba la fragancia de un saber adquirido por la investigación personal, de un aprender por propia experiencia (...) (Sainz, 19-20).

\footnotetext{
5 "La pregunta por lo que la escuela enseña nos lleva al territorio de lo que ella sabe, de lo que ella investiga y de lo que puede codificar para que sus alumnos aprendan" (García, 1996).

${ }^{6}$ Las referencias que en el presente apartado se hacen sobre el método de proyectos no lo retoman en su idea original, ni en las formas de aplicación que tuvo dentro del movimiento de Escuela Nueva: educación de los niños. Se accede a sus planteamientos generales de forma que esta sea una opción para la investigación dentro de la Escuela Normal Superior; se piensa en él desde la perspectiva de la investigación en el aula; se lo ve, también, como una forma de vincular la práctica pedagógica a los desarrollos de los núcleos y el equipo docente.
} 
La transformación de las prácticas de enseñanza en el movimiento de Escuela Nueva buscó la superación de los modelos tradicionales a través de una multiplicidad de métodos y experiencias, una de las cuales es el método de proyectos. No se trata de una novedad, en el marco de este movimiento, sino de un reordenamiento de ideas, nociones y conceptos en torno al niño y su actividad, a partir de los avances de la psicología y la biología. Estos pensamientos, de acuerdo con Luzuriaga (1964) se resumen en cinco ideas o principios: idea de la actividad, idea de la vitalidad, idea de la libertad, idea de la individualidad, idea de la colectividad. "Estas cinco ideas resumen la orientación general de la nueva educación, y se complementan y apoyan recíprocamente" (28).

Con estas ideas como base, la discusión pedagógica toma giros diferentes y se plantean métodos colectivos, métodos individuales, métodos de trabajo individual colectivo, métodos de trabajo por grupos, métodos de trabajo social, que buscan dar respuesta a la necesidad de una educación para la acción y para la vida. En este marco de interrogantes e innovaciones se inscribe el método de proyectos.

El método de proyectos es una opción para transformar la práctica de la Normal, para modificar las relaciones entre los núcleos en la medida en que se requiere su concurso para el desarrollo de los proyectos, pues se trata de alterar la cotidianidad para hacerla objeto de indagaciones y preguntas, para convertirla en lugar de acción y construcción. Debe quedar claro, entonces, que el método de proyectos funciona aquí como una alternativa didáctica, al lado de muchas otras que se pueden implementar, como una forma de práctica pedagógica, en la medida en que permite un modo de establecer la relación entre teoría y práctica; además, es una opción para superar el asignaturismo, en tanto su organización flexible y abierta requiere el apoyo de los núcleos y el equipo docente, pues el proyecto convoca la presencia de uno de los núcleos, elección hecha en virtud del énfasis adoptado por la Normal.

En este aspecto, si se quiere, hay una razón más, de orden cognitivo: el desarrollo del método de proyectos no avanza de manera secuencial; la lógica con la que se accede al conocimiento es la lógica de las relaciones que se establecen entre las temáticas, los temas o los contenidos. Se dota de muchos sentidos el espacio de relaciones conceptuales e intersubjetivas, de forma tal que se multiplican las voces, los caminos y las intenciones.

Una razón más para adoptar el método de proyectos como una opción didáctica tiene que ver con la multiplicación de las imágenes del maestro: éste se observa a sí mismo, en la experiencia de sí, como enseñante, como especialista y como sujeto de saber pedagógico.

En relación con los referentes formativos, el método de proyectos abre las puertas de la institución y la vincula con la ciencia, la cultura, la estética, el entorno. Así, logra sacar a la Normal del aislamiento en que se encuentra y ponerla en contacto con las realidades locales y regionales, de modo que se produce un equilibrio entre el interior y el exterior, el adentro y el afuera. Este proceso de reconocimiento del entorno ubica al maestro frente a sí mismo, en tanto le ponen en frente de condiciones, situaciones y contextos desde los cuales puede autocriticarse, revisarse y legitimarse, en el arduo y constante ejercicio de la experiencia.

$\mathrm{Si}$ se tienen en cuenta los conceptos articuladores como factores esenciales en la construcción del campo aplicado y como elementos a través de los cuales la Normal se apropia del presente a través de "un campo (...) con potencia de regulación, producción, traducción, prescripción y delimitación del flujo de conceptos, objetos y experiencias que lo afectan procedentes de la sociedad, la cultura y la ciencia" (Echeverri, 1996: 94), es posible encontrar en el método de proyectos un espacio propicio para que desde el núcleo 
se realicen reconceptualizaciones en la medida en que analiza los desplazamientos de los conceptos articuladores por los espacios de los saberes específicos, así como las experiencias de otros contextos y espacios.

En resumen, el método de proyectos remite a la presencia de lo experimental y experencial en el aula, de modo que hay una manifestación, una expresión del campo aplicado.

\section{Una definición}

El método de proyectos se define desde tres instancias: como estrategia pedagógica, como estrategia didáctica y como estrategia cognitiva.

El proyecto como estrategia pedagógica debe atender la forma en que la relación entre el maestro y el alumno se modifica en virtud de la estrategia didáctica. Alude a las formas, disposiciones, espacios y lugares en los que la experiencia de sí se despliega para transformar las percepciones, concepciones, sentidos y alternativas de la institución. En este proceso es necesario aclarar dos cosas: la primera tiene que ver con las formas de producirse que tienen los sujetos de la práctica (maestro y alumno) a partir del despliegue de su subjetividad, en el método de proyectos se especifica en las formas de expresar la enseñanza a través múltiples formas, lo que supone la apertura de las superficies del campo aplicado. En segundo lugar, la relación pedagógica dentro del dispositivo y desde la perspectiva del método de proyectos; ésta no se establece desde el alumno-maestro hacia lo individual, sino desde éste hacia el equipo docente, forma colegiada de la existencia del sujeto de saber pedagógico en la institución normalista.

También en el terreno de lo pedagógico, el método de proyectos debe referirse al espacio y el tiempo en la Escuela Normal. En el primero de los casos, es un asunto que se refiere no sólo al funcionamiento dentro de la institución; también considera la relación con los referentes formativos como se ha enunciado antes. En relación con el tiempo $-y$ como se ha venido planteando de acuerdo con el DFC-, se trata de romper el asignaturismo, de superar la dispersión en los procesos de formación y acceder a la conjugación de las propuestas e influencias en el terreno del campo aplicado. En la relación del tiempo con el método de proyectos, en lo que tiene que ver con la transformación de la jornada escolar, hay un primer nivel de relaciones, y es el que tiene que ver con la aplicación del método:

Caben varias soluciones transitorias pero nos fijaremos en tres: a) empleo del método de proyectos para algunas materias del programa solamente; b) uso de este método durante una o varias horas o en ciertos días de la semana; c) aplicación a un grado o sección de niños, los más adelantados por ejemplo (Sainz, 84-85).

Esto da la idea de que no se trata de una directriz institucional, o externa, que pretende la generalización del método de proyectos. Se trata más bien de una invitación a ensayar, a probar, y desde este proceso acceder a la experiencia, la experimentación, la apropiación y producción de conceptos y teorías acerca de la formación de maestros.

En la óptica del proyecto como estrategia pedagógica es posible añadir una precisión más. Es la que está relacionada con el tipo de proyectos que se puede implementar en la Escuela Normal. Para ello es necesario aludir a los tipos o clases de proyectos que se pueden organizar. Para este aspecto la clasificación es variada, y de acuerdo con Luzuriaga (1964), las múltiples clasificaciones no aportan nada al método, en cambio, sí sirve para presentar una idea acerca de la polivalencia de la propuesta. 


\begin{tabular}{|l|l|l|}
\hline Autor & Criterio & Nombre \\
\hline Luzuriaga & $\begin{array}{l}\text { Actividad } \\
\text { Materias } \\
\text { Edad }\end{array}$ & $\begin{array}{l}\text { Físicas, manuales, artísticas, intelectuales } \\
\text { Históricas, científicas, geográficas } \\
\text { Infantiles, mayores }\end{array}$ \\
\hline $\begin{array}{l}\text { Krackowitzer } \\
\text { Luzuriaga, } \\
1964)\end{array}$ & $\begin{array}{l}\text { Juego como objetivo } \\
\text { Actividades constructivas } \\
\text { Experiencia social }\end{array}$ & $\begin{array}{l}\text { Juego como finalidad, juego representativo, } \\
\text { juego dramático } \\
\text { Constructivas, } \\
\text { representaciones } \\
\text { La casa, higiene y confort, la comunidad, el } \\
\text { caserío, la escuela }\end{array}$ \\
\hline $\begin{array}{l}\text { Stevenson } \\
\text { Luzuriaga, } \\
1964)\end{array}$ & $\begin{array}{l}\text { Finalidad ética } \\
\text { Experiencia natural } \\
\text { Extividad }\end{array}$ & $\begin{array}{l}\text { Manuales, intelectuales } \\
\text { Simples, complejas }\end{array}$ \\
\hline Sainz & $\begin{array}{l}\text { Materias o disciplinas } \\
\text { Experiencia } \\
\text { proporcionan }\end{array}$ & $\begin{array}{l}\text { Idiomas, ciencias, artes, civismo } \\
\text { Cultivan la disciplina, el gusto estético, el } \\
\text { amor a la naturaleza, la afición al deporte, } \\
\text { los sentimientos éticos }\end{array}$ \\
\hline
\end{tabular}

Fuente: Lorenzo Luzuriaga. La educación nueva. Buenos Aires: Lozada, 1964.

También es necesario tener en cuenta el contenido del proyecto. En la idea de Kilpatrick (Luzuriaga, 1964) el surgimiento de los proyectos se da, básicamente, por el interés de los alumnos, "sólo los proyectos que surgen del interés espontáneo de los niños son utilizados" y con base en esto, hay quienes afirman que los proyectos planteados por los profesores están condenados al fracaso. Aunque no hay un consenso frente a quien debe proponer los proyectos y en qué momento, para la Escuela Normal Superior está situación queda salvada por el hecho de que ella debe proponer u organizar los proyectos que mejor se adapten a la vida institucional y al desarrollo del plan de estudios, pues es claro que en la Normal el proyecto tiene fines particulares, que se han referido antes. Por ello, "el proyecto en la escuela no echa mano del saber, ni de la técnica para obtener el producto, sino a la inversa, la escuela finge el proyecto para proporcionar el saber y la experiencia juntamente con otros resultados" (Sainz, 28). En esa medida, la propuesta del proyecto puede surgir del núcleo o el equipo docente, a partir del momento que analizan los objetos de enseñanza y su organización en el proceso de formación.

En el método de proyectos, tomado como una estrategia didáctica, esto es, como un campo que incluye diversos modos de presentar el conocimiento, se deben conjugar dos tipos de didáctica: la didáctica pedagógica, propia de los maestros y con una amplia tradición en la literatura, y la didáctica cognitiva, la que detentan los especialistas en el saber. No es suficiente con una o con otra, es necesario que se encuentre un punto de interacción entre ambas de manera que haya un enriquecimiento de la visión del maestro y por tanto del ejercicio de la enseñanza. La didáctica pedagógica nos da luces sobre el enseñar como un acontecer (Martínez, 1990) que tiene en su interior la palabra del maestro; en tanto que la didáctica cognitiva, permite concebir al alumno como un ser activo, como un sujeto que construye el conocimiento, para lo cual emplea diferente procesos y estrategias. De alguna manera, esta didáctica cognitiva, pensada en términos de procesos y estrategias, es específica; esto es, existe una para cada uno de los saberes escolares; en cambio la pedagógica es general, lo que hace de ésta un didáctica 
traductiva que se encarga de retomar los avances y aportes de la anterior para conjugarlos en un espacio creativo: la enseñanza.

Como se dijo antes, el método de proyectos no es más que una alternativa didáctica entre muchas, y en el terreno del campo aplicado se dibuja como una línea de trabajo. Por ello, es obvio que junto a éste se presentan otras opciones: experiencias desencadenantes, los trabajos cortos y las fichas autocorrectivas (Lacueva, 1997).

Las experiencias desencadenantes son actividades amplias e informales que buscan la familizarización del niño con las múltiples realidades del mundo, y pretenden despertar las inquietudes que poco a poco configuran la escuela centrada en la investigación. Los trabajos cortos se refieren a tareas breves, pero fructíferas desde la perspectiva investigativa, que propician encuentros del alumno con la indagación, la pregunta y la sospecha. Entre éstas y las otras se encuentran los orígenes de los proyectos. De otra parte, las fichas autocorrectivas, una idea original de Freinet (1975), dan la posibilidad de avanzar de acuerdo con su ritmo, en la consolidación de estructuras, conceptos y teorías.

Como estrategia cognitiva, el proyecto hace alusión al modo como deben conducirse las asignaturas. Se defiende la idea de lo cognitivo en relación con la enseñanza y no únicamente desde el aprendizaje. Esto significa que el maestro puede hacer uso de las formas que el estudiante emplea para la construcción del conocimiento, utilizar las estrategias cognitivas a favor de la enseñanza, para estructurar las actividades de forma significativa, abierta, múltiple, en relación con el maestro, la enseñanza y el carácter formativo de la Escuela Normal Superior.

Además, desde esta posición se establecen relaciones con el carácter comprensivo de la práctica en el sentido de lo cognitivo pedagógico. Se trata de los posibles efectos que sobre la forma de operar sobre el mundo tienen el alumno y el maestro, para que:

a) Posibiliten la presentación del conocimiento como una forma específica de argumentación $y$, así, se invoque el principio de enseñabilidad presente en la medida en que se pregunta por la enseñanza de las ciencias?.

b) Se determinen los procesos cognitivos que tienen una mayor participación en la construcción de ese conocimiento, en particular, de modo que se potencian de forma tal que la enseñanza se organice de acuerdo con la estructura del pensamiento.

c) Conociendo el modo en que se argumenta en cada saber, es posible que el profesor, conocedor de su estructura, contribuya al diseño o potenciación de estrategias de aprendizaje que fortalezcan la argumentación y la comprensión.

\section{Propuesta}

En el nivel operativo, se requiere que se cumplan ciertas condiciones para la implementación adecuada del método de proyectos. La primera está relacionada con la configuración de los núcleos y el despliegue del equipo docente. Si estas dos instancias colectivas no funcionan, resultaría muy difícil la operacionalización de la investigación en la institución, así como la producción de campos aplicados. La segunda condición es la selección de objetos de conocimiento y la construcción de objetos de enseñanza dentro de los núcleos. Este nivel de organización y actuación de los núcleos es fundamental para diseñar los proyectos. Es importante y necesario que se haya accedido a un análisis de la estructura fundamental -conceptual, teórica y experimental-de cada núcleo con el fin de configurar un mínimo de objetos de enseñanza que son básicos para la formación del alumno-maestro. De este modo, además, es más fácil establecer la colaboración entre el proyecto y los núcleos como fuentes primeras del conocimiento específico. 
También es clave tener en cuenta, como se señaló antes, que la implementación del método de proyectos requiere elegir el camino más adecuado para ello, se trata de reconocer cuáles objetos de conocimientos intra núcleo y entre núcleos son susceptibles de desarrollarse a través de este mecanismo, y así, elegir la mejor alternativa de construcción de proyectos: por horas, por núcleos, por objetos de enseñanza. El método de proyectos no debe excluir el uso de otro tipo de estrategias didácticas, herramientas metodológicas o técnicas. En la institución pueden existir múltiples formas de ejercer la enseñanza: la clase magistral, el taller, el seminario, la conferencia.

\section{Referencias Bibliográficas}

Carr, Wilfred y Kemmis, Stephen. Teoría crítica de la enseñanza. La investigación-acción en la formación del profesorado. Barcelona: Martínez-Roca, 1988.

Echeverri, Jesús Alberto. "Premisas conceptuales del dispositivo formativo comprensivo", en Revista Educación y Pedagogía, Vol. 8 (16), segundo semestre (1996): 71-105.

. El campo conceptual de la pedagogía: contribuciones. Inédito, materiales del proyecto Aciforma. Medellín, 2000.

Eisner, Elliot. El ojo ilustrado. Indagación cualitativa y mejora de la práctica educativa. Barcelona: Paidós, $1^{\text {a }}$ edición, 1998.

Elliot, J. El cambio educativo desde la investigación-acción. Madrid: Morata, 1993.

Freinet, Élise. La escuela Freinet. Los niños en un medio natural. Barcelona: Laia, 1975.

García P., Ernesto. "Maestros investigadores: el reto de profesionalización”, en Hacia el rescate de la pedagogía. Santa Fe de Bogotá: Asonen (1995): 70-84.

Lacueva, Aurora. "Retos y propuestas para una didáctica contextualizada y crítica", en Revista Educación y Pedagogía, Vol. 9 (18), mayo-agosto (1997): 41-82.

Luzuriaga, Lorenzo. La educación nueva. Buenos Aires: Losada, $7^{a}$ edición, 1964.

Martínez Boom, Alberto. "La enseñanza como posibilidad del pensamiento", en Díaz, Mario y Muñoz, José (eds.). Pedagogía, discurso y poder. Bogotá: Corprodic. A (1990):151-173.

Palacio, Victoria, y Salinas, Marta Lorena. "El campo aplicado, urdimbre para la formación de maestros"; en Revista Educación y Pedagogía, Vol. 8 (16), segundo semestre (1996):106-125.

Sainz, Fernando. El método de proyectos. Buenos Aires: Losada, $3^{a}$ edición, 1951.

Vasco, Eloísa. Maestros, alumnos y saberes. Santa Fe de Bogotá: Cooperativa Editorial del Magisterio, Colección Mesa Redonda, 1995. 